\title{
Linguistic description and analysis
}

92-436 Dryer, Matthew S. (State U. of New York at Buffalo). The Greenbergian word order correlations. Language (Baltimore, Md), 68, 1 (1992), 81-138.

This paper reports on the results of a detailed empirical study of word order correlations, based on a sample of 625 languages. The primary result is a determination of exactly what pairs of elements correlate in order with the verb and object. Some pairs of clements that have been claimed to correlate in order with the verb and object do not in fact exhibit any correlation. The author argues against the Head-Dependent Theory (HDT), according to which the correlations reflect a tendency towards consistent ordering of heads and dependents. He offers an alternative account, the Branching Direction Theory (BDT), based on consistent ordering of phrasal and nonphrasal elements. According to the BDT, the word order correlations reflect a tendency for languages to be consistently rightbranching or consistently left-branching.

\section{Phonetics and phonology}

92-437 Enomoto, Kayoko (U. of Edinburgh). Interlanguage phonology: the perceptual development of durational contrasts by English-speaking learners of Japanese. Edinburgh Working Papers in Applied Linguistics (Edinburgh), 3 (1992), 25-35.

This paper reports upon a small-scale pilot study for further investigation into the perceptual development in the acquisition of durational contrasts by different levels of adult English-speakers of Japanese. Four kinds of naturally spoken word-tokens were edited by a synthesiser to generate word-stimuli varying the duration of a certain consonant/vowel (originally contained in each word-token) along a durational continuum. These synthetic word-stimuli were randomised and presented to both native speakers and English-speaking learners of Japanese, in four forced-choice word identification tasks. The

overall identification data in all tasks, indicated that the L2 learners' levels of language experience/ proficiency correlated positively with the closeness of their perceptual categories of the durational continua, to the perceptual categorisation of the native speaker group. This seems to imply that the L2 learners' initial perceptual categories or perceptual targets, may be possibly modified/ developed towards the 'categorical perception' model shown by the native speakers in the course of adult $\mathrm{L} 2$ acquisition.

\section{2-438 Hallé, Pierre A. and others. Beginnings of prosodic organisation:} intonation and duration patterns of disyllables produced by Japanese and French infants. Language and Speech (Hampton Hill, Middx), 34, 4 (1991), 299-318.

In this study, some prosodic aspects of the disyllabic vocalisations (both babbling and words) produced by four French and four Japanese children of about 18 months of age, are examined. $F_{0}$ contour and vowel durations in disyllables are found to be clearly language-specific. For French infants, rising $\mathrm{F}_{0}$ contours and final syllable lengthening are the rule, whereas falling $F_{0}$ contours and absence of final lengthening are the rule for Japanese children. These results are congruent and adult prosody in the two languages. They hold for both babbling and utterances identified as words. The disyllables produced by the Japanese infants reflect adult forms not only in terms of global intonation patterns, but also in terms of tone and duration characteristics at the lexical level. 
92-439 Levitt, Andrea G. (Haskins Labs. and Wellesley Coll.) and Qi Wang (Haskins Labs. and $U$. of Connecticut). Evidence for language-specific rhythmic influences in the reduplicative babbling of French-and English-learning infants. Language and Speech (Hampton Hill, Middx), 34, 3 (1991), 235-49.

The reduplicative babbling of five French- and five English-learning infants was examined for evidence of language-specific rhythmic patterns. Three types of patterns were examined: final-syllable lengthening, timing of nonfinal syllables, and number of syllables per utterance. Both English and French exhibit final-syllable lengthening but, because of variable word stress, English tends to lengthen nonfinal stressed syllables, as well as final ones. Thus French final-syllable lengthening is more salient because it is the only regular source of lengthening. There is also some indication that the magnitude of final syllable lengthening is greater in French than in English. In addition, French nonfinal syllables, which are not subject to English variable word stress, are generally equal in length, whereas English tends to have more irregularly timed nonfinal syllables. Finally, French breath-groups often contain as many as four to six syllables, whereas intervals between stressed syllables in English rarely contain more than three syllables. The reduplicative babbling of the French and American infants showed characteristics of the ambient language. In particular, the babbling of the French infants showed a significantly higher percentage of final-syllable lengthening than that of the American infants. The magnitude of their final-syllable lengthening was also greater. The French infants also produced significantly more reduplicative babbles that were four or more syllables in length. Finally, the nonfinal syllables of the French infants tended to be more regularly timed than those of the American infants, although this pattern emerged only in the later stage of the infants' reduplicative babbles.

\section{2-440 Schuetze-Coburn, Stephan and others (U. of California at Los} Angeles). Units of intonation in discourse: a comparison of acoustic and auditory analyses. Language and Speech (Hampton Hill, Middx). 34, 3 (1991), 207-34.

Measurements were made of the correspondence between acoustic (declination) units and auditory (intonation) units in American English conversation in an attempt to relate these two levels of description. The results showed that 99 per cent of the acoustic unit boundaries coincided with auditory unit boundaries, indicating that the acoustic cues of $F_{0}$ reset, pause, and speaker change used in defining the acoustic units were perceived, verifying the validity of both types of analyses. However, the units differed in scope, with many more auditory units occurring than acoustic units. This result suggests that the common practice of assuming the 'intonational phrase' to be the primary domain of prosodic phenomena such as declination does not adequately account for the authors' data of larger periods of speech. Both acoustic and auditory analysis are needed for a satisfactory description of intonation. The establishment of a methodological connecting link between the acoustic and auditory dimensions suggests that further comparisons of such different data types may yield guidelines for regularising the relationship between the two levels.

\section{Sociolinguistics}

\section{2-441 Bamiro, Edmund 0. (U. of Maiduguri, Nigeria). The social and functional power of Nigerian English. World Englishes (Oxford), 10, 3 (1991), 275-86.}

This paper employs the frameworks of sociolinguistics and social psychology to explore the social and functional power of Nigerian Pidgin English (NPE) as it is featured in the novels of two prominent Nigerian authors, Wole Soyinka and Chinua Achebe. It is argued that, although NPE has not risen to the position of the acrolect or the prestige variety, its social and functional power as an interpreter of the Nigerian social structure cannot be discounted. Thus, the analyses undertaken in this study not only demonstrate how NPE exposes the tension and conflict between the dominant and dominated groups in Nigeria, but also how it reveals the contempt and anger the dominated have for the dominant. NPE thus becomes a code that symbolises the solidarity of the dominated class in Nigeria. Furthermore, the analyses not only demonstrate how the dominant class use NPE as an accommodation strategy, but also how some privileged members of the dominated groups employ NPE to maintain their communicative and social distance, thereby enforcing power relations and 
neutralising cultural norms. The analyses also show how the codes of the dominated groups in Nigeria become the vehicle by which they are further exploited and subjugated by the dominant class.

\section{2-442 Camilleri, Antoinette (U. of Edinburgh). The sociolinguistic status of English in Malta. Edinburgh Working Papers in Applied Linguistics (Edinburgh), 3 (1992), 14-24.}

This paper provides a sociolinguistic description of English language use in Malta at present, in terms of who speaks what language, where and when. Some observations on English language contact with
Maltese are made. Finally a brief discussion is taken up as to whether and to what extent it would be appropriate to consider Maltese English as a new institutionalised variety of English.

\section{2-443 Edwards, Walter F. (Wayne State U., Detroit, MI). Sociolinguistic} behaviour in a Detroit inner-city black neighbourhood. Language in Society (Cambridge), 21, 1 (1992), 93-115.

The integration of 66 black Detroit inner-city residents (33 males and 33 females) into their neighbourhood is measured quantitatively by a Vernacular Culture Index (VCI) constructed from the respondents' responses to 10 statements, each with values ranging from 1 to 4 . The speech behaviours of these respondents with respect to variants of six linguistic variables (each with a black English [BE] and a colloquial Standard English variant) are quantified and the statistical relationships between the VCI scores and linguistic behaviour are determined. The results of the study reveal that respondents in the older age groups $(40-59,60+)$ are more likely to choose $\mathrm{BE}$ variants than respondents in the younger age groups (18-25, 26-39), and that there are consistent statistically significant correlations between high scores on the VCI and the choice of BE variants of the linguistic variables. This is taken to support the proposal that the Social Network Theory approach, of which the VCI concept is a part, is capable of explaining intracommunity linguistic variation in socioeconomically homogeneous areas such as the black community studied.

\section{2-444 Kristiansen, Tore ( $U$. of Copenhagen) and others Ethnolinguistic vitality in 'the Danish capital of America'. Journal of Multilingual and Multicultural Development (Clevedon, Avon), 12, 6 (1991): 421-48.}

This paper analyses objective/subjective and qualitative/quantitative aspects of vitality in Solvang, a Danish-American community. The setting is interesting for its radical transformation from being all about leading a fulfilling (Grundtvigian) community life to being instrumentally all about attracting tourists with an external Danish facade. By ethnographic-like means, analyse the historical vitality of Solvang to the present leading us to formulate hypotheses for testing in three investigations using the Subjective Vitality Questionnaire (SVQ) with adolescent and adult Danish- and Anglo-Americans. Informants were realistic in their evaluations associating Danish strengths with the past, judging Anglos and Danes as currently equal economically and culturally, and ranking Danes lower than both Anglos and Hispanics as to language use and demographics. Younger Anglos viewed Hispanic vitality more positively than their elders. A so-called 'perceptual distortions in favour of the outgroup' profile emerged, such that Anglos perceived Danish vitality higher than the latter themselves, whereas Danes construed Anglo vitality higher than did Anglos. Finally, whether Hispanics were included in the judgemental frame or not had significant effects on the ratings of Anglo and Danish vitalities, findings having telling implications for future research. The value of adopting truly ethnographic and quantitative analyses of vitality in tandem is underscored. 
92-445 Lie, Suzanne and Wold, Astri Heen (U. of Oslo, Norway). Linguistic minority children's comprehension of language in the classroom and teachers' adjustment to their pupils' performance: a Norwegian case study. Journal of Multilingual and Multicultural Development (Clevedon, Avon), 12, 5 (1991), 363-382.

This exploratory study is an attempt to examine linguistic minority (LM) children's comprehension of the language used by the teacher in a fifth grade classroom of Norwegian and LM children. A further goal is to examine how teachers adjust their communication to their pupils' language competence. A language comprehension test was developed on the basis of two hours of recorded instruction on local history by the teacher. Both Norwegian and LM children were tested individually. A number of teachers were asked to evaluate the expected difficulty of the same test items for both groups. The results show that some of the LM children still have serious comprehension problems despite the fact that they were born in Norway and had all their schooling within the Norwegian school system. Words related to the physical context of the classroom and frequently used abstract expressions were well understood. Words less frequently used posed problems for the weakest pupils even in cases where the words were concrete and understanding context-dependent. The teachers correctly expected the LM children to comprehend less than their Norwegian classmates. However, they systematically accentuated the differences between pupil groups.

\section{2-446 Lüdi, Georges. Internal migrants in a multilingual country. Multilingua} (Amsterdam), 11, 1 (1992), 45-73.

This paper presents results of a research project on linguistic aspects of migration across the language border in Switzerland. The focus is on Frenchspeaking migrants living in Basle and Berne, two cities characterised by a German/Swiss German diglossia. In order to answer the question of how migrants experience the redefinition of their identity (what options do they have in this respect? what role does the diglossia play in this process?) various quantitative and qualitative approaches have been combined. The dialect, considered in the Romandie to be the main communicative stumbling block in dealing with German-speaking Switzerland, is shown to be spontaneously acquired by most migrants and represents an important symbol of integration. The reading of the main identity markers then reveals the existence of several identity options which are correlatable with communicative practices within the migrants' communicative networks, the use of the dialect assuming the value of an 'act of identity'. It can be shown that francophones in Basle and Berne do not constitute a 'community' in the classical sense, but that prototypes of migrants emerge according to the communicative environment which represent different solutions that are worked out, tested and improved in social interaction.

\section{2-447 Lyon, Jean and Ellis, Nick (University Coll. of North Wales). Parental attitudes towards the Welsh language. Journal of Multilingual and Multicultural Development (Clevedon, Avon), 12, 4 (1991), 239-51.}

Two main reasons have been suggested for learning or for retaining a language: utility and esteem. The Welsh language is still declining. Reasons for this decline could lie in the attitudes of Welsh and nonWelsh speakers towards the language. As legislation has attempted to facilitate the use of Welsh, opinions about the language were examined. Subjects comprised groups of parents from differing English/ Welsh speaking backgrounds.

An overall favourable attitude towards Welsh was found in all groups, with 86 per cent of parents wanting their children to learn Welsh. The reasons given most often for this support related to Welsh identity. Other reasons included better communication and enhancement of employment prospects. Most parents also expected Welsh to be used as much in the future as at present.

Language has been closely associated with cultural identity but attitudes to the language and its future did not map neatly onto language background groups. However, for Welsh speaking parents, cultural reasons are of greatest importance, whereas English speaking parents are motivated by practical considerations. 


\section{2-448 McGregor, Graham (U. of Otago, Dunedin, New Zealand) and Wei Li}

(U. of Newcastle upon Tyne). Chinese or English? Language choice amongst

Chinese students in Newcastle upon Tyne. Journal of Multilingual and Multicultural

Development (Clevedon, Avon), 12, 6 (1991), 493-509.

The demography and patterning of multilingualism and multiculturalism in Britain has yet to be fully explored. This neglect is especially evident in the case of the Chinese community. Exploration of the ethnographic background of this community by origin, social background, language and generation reveals the extent of its linguistic and social diversity.

As a first step towards investigating the differential social experiences and language development of subgroups of Chinese in the UK, findings from a study of language choice amongst a group of Chinese students are presented. Elicited information on language choice in different situations and language attitudes reveals that the most salient influence on speakers' linguistic behaviour comes from the identity of the audience, with factors such as topic and setting subservient to particular audience types [examples with discussion]. This finding supports Bell's (1984) theory of audience design, which highlights the sociocultural identity and the interpersonal relationship of the participants as an important dimension of language variation and choice. Furthermore, it stresses the need for any investigation into the internal structuring and cultural values of communities to be sensitive to their social and behavioural background; thus we might hope to work towards enhancing interethnic communication in a multicultural context.

92-449 Milroy, Lesley and Milroy, James ( $U$. of Newcastle upon Tyne). Social network and social class: toward an integrated sociolinguistic model. Language in Society (London). 21, 1 (1992), 1-26.

In sociolinguistics, approaches that use the variables of socioeconomic class and social network have often been thought to be irreconcilable. In this article, the authors explore the connection between these variables and suggest the outlines of a model that can integrate them in a coherent way. This depends on linking a consensus-based microlevel of network with a conflict-based macrolevel of social class. The authors suggest interpretations of certain sociolinguistic findings, citing detailed evidence from research in Northern Ireland and Philadelphia, which emphasise the need for acknowledging the importance of looseknit network ties in facilitating linguistic innovations. They then propose that the link between network and class can be made via the notion of weak network ties using the process-based model of the macrolevel suggested by Thomas Højrup's theory of life-modes.

\section{2-450 Myers-Scotton, Carol. Constructing the frame in intrasentential codeswitching. Multilingua (Amsterdam), 11, 1 (1992), 101-27.}

This paper presents a model to explain structural constraints on code-switching. A major claim is that codeswitching, like other naturally-occurring language data, follows principles which are internally present as part of the speaker's grammatical competence. The model relies crucially on making a distinction in codeswitching between the matrix language, which has the dominant role, and the embedded language(s). It also explains codeswitching data in terms of a distinction between content and system morphemes. Empirical data support these claims.

\section{9-451 Nicholson, Rangi and Garland, Ron (Massey U., New Zealand). New} Zealanders' attitudes to the revitalisation of the Maori language. Journal of Multilingual and Multicultural Development (Clevedon, Avon), 12, 5 (1991). 393-410.

While Maori language revitalisation efforts intensified in the 1970s, and achievements to date seem impressive, by the end of the 1980 s there were probably 10,000 fewer fluent speakers of Maori than at the end of the previous decade. With many fluent Maori speakers aged over 55, further promotion of the Maori language is essential and urgent if Maori is not to remain as an endangered language.

This paper reports the results of a nationwide mail survey of 225 New Zealand adults' opinions about the Maori language's role in contemporary society and the extent to which New Zealanders 
will commit themselves to fostering the language. While two thirds of respondents felt the language had a place in New Zealand society, only one quarter of the sample indicated that the language should be used to a greater extent than now. Bilingualism in Government agencies, bilingual signs and bilingual product labelling received minimal support. However, Maori respondents, younger people and women favoured bilingualism more than their European, older and male counterparts.

The paper confirms the claims of Spolsky (1989) that the basis exists for revitalisation of the Maori language. It also draws upon the marketing paradigm (Cooper, 1985) for promoting the use of Maori language in the home, in the neighbourhood, in the community and beyond (cf. Fishman, 1990).

\section{2-452 Roberts, Alasdair (Northern Coll., Aberdeen). Parental attitudes to Gaelic-medium education in the Western Isles of Scotland. Journal of Multilingual and Multicultural Development (Clevedon, Avon), 12, 4 (1991), 253-69.}

Gaelic-medium education is an optional extension of the bilingual policy which has been applied to all schools of the Western Isles during the 1980s. The bilingual policy is that Gaelic-speaking children will be as literate and fluent in Gaelic as in English by P7, and that provision will be made at all stages of primary education for English-speaking children to learn Gaelic as a second language in accordance with the wishes of their parents. The first Gaelic-medium unit teaching school entrants through Gaelic across the curriculum opened in 1986 and the number of such units within primary schools has since risen to 11. This questionnaire and interview survey shows that Gaelic is no longer the language of the home for a majority of parents of pre-school children, who are nevertheless very positive that their children should be bilingual by the end of primary school. About half seemed to be prepared to enrol their children in Gaelic-medium units if these were provided, although thee was a degree of uncertainty about the nature of Gaelic-medium education as distinct from bilingual education.

\section{2-453 Schiffman, Harold F. (U. of Washington). 'Resisting arrest' in status planning: structural and covert impediments to status change. Language and Communication (Oxford), 12, 1 (1992), 1-15.}

Attempts at language status planning in postcolonial societies, whereby an exogenous language such as English or French is suppressed and an indigenous language upgraded to take its place, have often failed. It is argued that the resistance to status planning is not due to external factors but is probably the result of past and current language policies per se. Such a situation is evident in India, where English predominates because of its high functional load as the internationally recognised language of business, higher education and the law; there are no indigenous registers which could act as substitutes in these domains. Similarly, English in Malaysia and High German in Switzerland may never be replaced as legal registers, if planners continue to ignore both how such registers come into existence and their functional load. Even countries with no officially sanctioned language may operate a covert policy, such as the United States, which overwhelmingly favours English in all spheres of activity. It may be that for an increased understanding of status change and implementation, the most fruitful investigations may lie in the area of covert language policy.

\section{2-454 White, Michael J. and Yan Li (Ball State U.. In). Second-language fluency and person perception in China and the United States. Journal of Language and Social Psychology (Clevedon, Avon), 10, 2 (1991), 99-113.}

The hypothesis that fluency of non-native speakers would affect how they were perceived by native speakers was examined. Speech samples were first collected from non-native speakers of Chinese and from non-native speakers of English. Each language sample was then rated for fluency, after which 110 native Chinese speakers and 114 native American English speakers rated the non-native speakers of the respective languages on seven personality scales. A multivariate analysis showed that more fluent nonnative speakers of either language were perceived more positively by native speaking raters than less fluent speakers. Ratings were influenced by the order in which raters heard speakers. This was manifested by a main effect for order among American raters, and an interaction effect with fluency for both groups. The fluency of initially heard speakers appeared to establish a standard 
against which subsequently heard speakers were judged. These findings were observed using personality descriptors which have similar connotative and denotative meaning across the two languages, allowing comparability of results. Although findings were very similar for both groups, increases in fluency affected ratings more uniformly for Chinese than for American raters. Implications for contextual influences on person perception and social cognition are discussed.

\section{Psycholinguistics}

92-455 Blake, Joanna (York U., Ontario. Canada) and de Boysson-Bardies, Bénédicte (CNRS). Patterns in babbling: a cross-linguistic study. Journal of Child Language (Cambridge), 19, 1 (1992), 51-74.

Three Canadian-English infants and three ParisianFrench infants were filmed bi-weekly for three to five months, from the age of $0 ; 9$ or $0 ; 11$ until $1 ; 2$, at home in naturalistic interaction with a parent. Their babbled utterances were transcribed phonetically and categorised according to consonanttype and vowel-type. The contexts for each utterance were described in terms in both specific and more general contextual categories. Observed frequencies of co-occurrences between phonetic and contextual categories were compared to expected frequencies, and deviations were considered to be patterns in babbling. Patterning increased after age $1 ; 0$ for most infants, and from 15 per cent to 30 per cent of each infant's babbled utterances recurred in particular contexts with a greater-than-expected frequency. Similarities in patterns were found both within and across language groups. These soundmeaning correspondences in babbling are viewed as continuous with early situation-bound meaning in words.

92-456 Dart, Sarah N. (U. of California at Los Angeles). Narrative style in the two languages of a bilingual child. Child Language (Cambridge), 19, 2 (1992), 367-87.

This study involves the analysis of a kind of data not often found in the literature: spontaneous storytelling without an audience and therefore free of any direct influence from another person. The stories are told by a bilingual four-year-old, with examples from both English and French. In comparing the data from both languages, it was found that the French narratives contained a much larger percentage of modifiers (adjectives, adverbs and relative clauses) and employed a greater variety of tenses, which were manipulated to highlight various parts of the story. Comparison with data from children's books in the two languages largely supports the interlinguistic differences in the oral data.

\section{2-457 Letts, Carolyn A. (U. of Reading). Early second language acquisition: a comparison of the linguistic output of a pre-school child acquiring English as a second language with that of a monolingual peer. British Journal of Disorders of Communication (London), 26, 2 (1991) 219-34.}

Two pre-school children were recorded at regular intervals over a 9-month period while playing freely together. One child was acquiring English as a second language, whilst the other was a monolingual English speaker. The sociolinguistic domain was such that the children were likely to be motivated to communicate with each other in English. A variety of quantitative measures were taken from the transcribed data, including measures of utterance type, length, type-token ratios, use of auxiliaries and morphology. The child for whom
English was a second language was found to be well able to interact on equal terms with his partner, despite being somewhat less advanced in some aspects of English language development by the end of the sampling period. Whilst he appeared to be consolidating his language skills during this time, his monolingual partner appeared to be developing rapidly. It is hoped that normative longitudinal data of this kind will be of use in the accurate assessment of children from dual language backgrounds, who may be referred for speech and language therapy. 
92-458 Levitt, Andrea G. and Utman, Jennifer G. Aydelott (Haskins Labs. and Wellesley Coll.) From babbling towards the sound systems of English and French: a longitudinal two-case study. Journal of Child Language (Cambridge), 19, 1 (1992). 19-49.

The utterances of one French and one American infant at $0 ; 5,0 ; 8,0 ; 11$, and $1 ; 2$ were transcribed and acoustically analysed for syllable duration and vowel formant values. Both general and languagespecific effects emerged in the longitudinal study. Initial similarities in the consonantal repertoires of both infants, increasing control in producing target $F_{1}$ and $F_{2}$ values, and developmental changes in babbling characteristics over time seem to reflect universal patterns. Yet the babbling of the infants differed in ways that appear to be due to differences in their language environments. Shifts in the infants' sound repertoires reflected phoneme frequencies in the adult languages. The English-learning infant produced more closed syllables, which is characteristic of English, than the French-learning infant. The French-learning infant tended to produce more regularly-timed nonfinal syllables and showed significantly more final-syllable lengthening (both characteristic of French) than the English-learning infant.

92-459 Mannle, Sara and others (Emory U., GA). Two-year-olds' conversations with their mothers and preschool-aged siblings. First Language (Chalfont St Giles. Bucks), 12, 1 (1991), 57-71.

The pragmatics of sibling-infant and mother-infant conversations were compared. Sixteen children, 22 to 26 months of age, were videotaped for 15 minutes in dyadic interaction with their mothers and for 15 minutes in dyadic interaction with their preschool-aged siblings. Sibling-infant and mother-infant conversations were compared on three dimensions: quantitative characteristics, conversational style and conversational repair of potential breakdowns. Compared with mothers and infants, siblings and infants talked less and had shorter conversations. On an individual level, siblings asked fewer questions of the infants and issued more directives to them than did the mothers.
Moreover, siblings failed to repair disruptions in conversations almost twice as often as mothers. The infants' conversational behaviours, however, did not differ when interacting with the siblings as opposed to the mothers. These results indicate that preschool-aged siblings are not yet adept at making the kinds of pragmatic adjustments in their speech that scaffold infants in their early conversational interactions. It is suggested that the experience laterborn infants have with less responsive siblings may be valuable preparation for interacting with strangers, especially peers, who share many characteristics with siblings.

\section{2-460 Obler, Loraine K. (City U. of New York Graduate Sch./Emerson Coll.) and others. Auditory comprehension and aging: decline in syntactic processing. Applied Psycholinguistics (Cambridge), 12, 4 (1991), 433-52.}

Comprehension of six syntactic structures was tested across four age groups. Each structure was presented with both plausible and implausible content. The contribution of cognitive nonlinguistic factors important for comprehension (attention, short-term memory, and mental control) was tested via standard neuropsychological tasks. Sixty-six women aged 30-79 were tested. Both errors and reaction times increased with age, especially for more complex syntactic types and implausible sentences. The neuropsychological factors tested contributed minimally to an age-related decline in comprehension, suggesting that the subtle breakdown seen in syntactic processing may be a language-specific impairment. 
92-461 Papoušek, Mechthild (Max-Planck Inst. for Psychiatry, Munich) and Shu-Fen C. Hwang (NICHD, Bethesda). Tone and intonation in Mandarin babytalk to presyllabic infants: comparison with registers of adult conversation and foreign language instruction. Applied Psycholinguistics (Cambridge) 12, 4 (1991), 481-504.

Six native speakers of Mandarin Chinese recorded 140 preselected utterances in three role-play contexts that differentially elicited registers of babytalk to presyllabic infants (BTP), foreign language instruction (FLI), and adult conversation (AC). Sound spectrograms were used to obtain 10 measures of fundamental frequency ( $F o$ ) patterns for comparisons among the three registers. In FLI, the speakers expanded Fo patterns in time and Fo range in comparison with AC. They clarified lexical tonal information and seemed to reduce suprasegmental information. In BTP, the speakers raised peak and minimum Fo, reduced the rate of Fo fluctuations, and increased the proportion of terminal rising contours. The speakers reduced, neglected, or modified lexical tonal information in favour of simplified and clarified intonation contours. The significance of the results is discussed in relation to tone acquisition in children and to a universal intuitive didactic competence in caretakers.

\section{2-462 Paul, Rhea (Portland State U.) and Shiffer, Mary E. (North Clackamas} School District). Communicative initiations in normal and late-talking toddlers. Applied Psycholinguistics (Cambridge), 12, 4 (1991), 419-31.

Initiation of communication in videotaped, unstructured mother-child interactions was examined in two groups of 2-year-olds: those with normal language development and those with late acquisition of expressive language. Results revealed that the late talkers (LTs) expressed significantly fewer intentions, but that the difference between the two groups could be accounted for entirely by the difference in one type of intention: the expression of joint attentional intensions. Investigation of the forms of expression of intentions showed that the normal group used significantly more verbal forms of expression, as expected. The predominant form for the normal group was word combinations, while the predominant form for the LTs was vocalisation. The implications of these results for understanding the mechanisms involved in early language delay are discussed.

\section{2-463 Pérez-Pereira, M. and Castro, J. (U. of Santiago de Compostela.} Spain). Pragmatic functions of blind and sighted children's language: a twin case study. First Language (Chalfont St Giles, Bucks), 12, 1 (1992), 17-37.

Results are presented from a longitudinal study on the acquisition of Spanish by twin girls, one of whom was blind, the other of whom was sighted. Analyses were undertaken of the MLU of the subjects from the age of $2 ; 5$ to $3 ; 5$, and on the functional categories used by both children. The data indicate that (1) the blind girl had a slightly higher MLU than her sighted sister, and (2) the two girls exhibited differences in their pragmatic uses of language. While the blind girl used language for referring to her own actions, with more routines, calls and repetitions, the sighted child tended to use language in a way which was more bound up with external reality and social interchanges. Both children seemed to have different strategies for learning language, the blind one being closer to the Gestalt type.

\section{2-464 Tyler, Ann A. (U. of Nevada, Reno) and Saxman, John H. (Syracuse U.). Initial voicing contrast acquisition in normal and phonologically disordered children. Applied Psycholinguistics (Cambridge), 12, 4 (1991), 453-79.}

Speech sound production changes that occurred during acquisition of the initial voicing contrast induced through treatment in phonologically disordered children were compared to those that occurred during normal acquisition of the contrast. Target stop productions from three normally developing children and six phonologically dis- ordered children were analysed acoustically along the temporal measure of VOT. The phonologically disordered subjects displayed different patterns of voicing contrast acquisition, none of which replicated the normally developing children's pattern. The phonologically disordered children's patterns of acquisition were characterised by larger 
and more variable VOT values in comparison to those of the normally developing children. Different patterns of voicing contrast acquisition observed in
VOTs are discussed with regard to the nature of linguistic change and the source of breakdown in children's speech sound errors.

\section{2-465 Whalen, D. H. (Haskins Labs.) and others. Intonational differences between the reduplicative babbling of French- and English-learning infants. Journal of Child Language (Cambridge), 18, 3 (1991), 501-16.}

The two- and three-syllable reduplicative babbling of five French-learning and five English-learning infants $(0 ; 5$ to $1 ; 1)$ was examined in two ways for intonational differences. The first measure was a categorisation into one of five categories (rising, falling, rise-fall, fall-rise, level) by expert listeners. The second was the fundamental frequency $\left(F_{0}\right)$ from the early, middle and late portion of each syllable. Both measures showed significant differences between the two language groups, 65 per cent of the utterances from both groups were classified as either rising or falling. For the French children, these were divided equally into the rising and the falling categories, while 75 per cent of those utterances for the English children were judged to have falling intonation. Proportions of the other three categories were not significantly different by language environment. In both languages, though, three-syllable utterances were more likely to have a complex contour than two-syllable ones. Analysis of the $F_{0}$ patterns confirmed the perceptual assessment. Several aspects of the target languages help explain these intonational differences in prelinguistic babbling.

\section{Pragmatics}

\section{2-466 Fries, Peter H. (Central Michigan U.). Lexico-grammatical patterns and the interpretation of texts. Discourse Processes (Norwood, NJ), 15, 1 (1992), 73-91}

It is well known that the meanings of sentences are affected by the contexts in which they are perceived. Texts create patterns of meaning which affect how the component sentences are interpreted in context. A careful study of a two-paragraph text which contains a high concentration of matching relations shows one technique by which this process happens. Repetitions of the experiential relations expressed in the text, together with the matching conjunctive relations which hold between the various messages of the text, establish paradigmatic and syntagmatic patterns in the meanings of the text which encourage readers to interpret the text (and its parts) in certain ways. These patterns also relate to dynamic aspects of the text, such as the use of denials, partial denials, and the probable placement of tonic accent. The technique of analysis proposed here may be extended to examine matching between large portions of a text (as in episodes of a story).

\section{2-467 Hartford, Beverly S. and Bardovi-Harlig, Kathleen (Indiana U.). Closing the conversation: evidence from the academic advising session. Discourse Processes (New York) 15, 1 (1992), 93-116.}

This article provides evidence that institutional conversations differ from natural conversations, even in their closings, an aspect of structure which they share. Utilising 31 academic advising session interviews, the authors contrast felicitous closings by both native and highly proficient non-native speakers with infelicitous closing by non-natives. They show that the closings of these interviews cannot be reopened in the ways described by Schegloff and Sacks (1973). In fact, the data show that native speaker interviews are not reopened at all, but are only followed by separate and highly limited post-session conversations. Since non-native speakers often do not know or follow the contextspecific constraints, their conversations provide evidence for the rules of such exchanges.

\section{2-468 Johnson, Donna M. (U. of Arizona). Compliments and politeness in peer-review texts. Applied Linguistics (Oxford), 13, 1 (1992), 51-71.}

This paper reports on an empirical analysis of the forms, strategies, and functions of complimenting in one genre of written discourse. The data base is a set of 51 peer-review texts written in an academic 
setting. First, analyses are provided of the syntactic and lexical patterns of 256 compliments. These analyses reveal the degree and nature of formulaicity in the compliments. A related discussion of politeness considerations in the use of syntactic framing strategies is also offered. Next, several complimenting discourse strategies used by writers to construct their texts are identified. It is found, for example, that writers exhibit a remarkable regularity in the use of opening compliments. It is argued that writers use complimenting discourse strategies to establish and maintain rapport and to mitigate both global and specific face-threatening acts and that these social purposes help to account for both their frequency and patterning in the texts.

92-469 Johnson, Donna M. and Roen, Duane H. (U. of Arizona).

Complimenting and involvement in peer reviews: gender variation. Language in Society (London), 21, 1 (1992), 27-57.

This article presents an analysis of gender differences in the use of compliments in one genre of written discourse. The data base is a set of 47 peer reviews of academic papers written by graduate students in the form of letters. Drawing on work from several theoretical perspectives, the authors analysed the forms, strategies, and discourse functions of compliments in these papers. It was found that women made significantly greater use of compliment intensifiers and personal referencing than did men.
In using compliments to structure discourse, women more often framed the text with both opening and closing compliments. Consistent patterns suggested that women writers accommodated to the gender of their addressee more than did men, resulting in a discernible female-female complimenting style. The study illustrates specific ways that gender is involved in being polite and in creating and sustaining a sense of involvement in written discourse.

\section{2-470 Kachru, Yamuna (U. of Illinois). Speech acts in world Englishes: toward} a framework for research. World Englishes (Oxford), 10, 3 (1991), 299-306.

Recent research has cast doubt on the adequacy, and thus universality, of certain pragmatic notions such as Gricean implicature and face. The inability of classical speech act theory to account for crosscultural interaction leads the author to propose a framework for research into speech acts in indigenous varieties of English.

It is argued that literature is a valuable source of data for such work in that it simulates actual conversations in authentic settings. This is supported by an examination of excerpts from four Indian English novels. Instances of leave-taking in a variety of contexts are discussed. Particular attention is given to the identification of culture-specific speech acts, such as the role of blessings in leave-taking and the use of kinship terms as terms of address for showing deference and solidarity.

Such accounts reveal the shortcomings of traditional analytic methods and appeal is made to a richer methodology which would incorporate notions used in speech act theory, conversational analysis, sociolinguistics and ethnography of communication.

\section{2-471 Ochs, Elinor (U. of California, LA) and others. Storytelling as a theory- building activity. Discourse Processes (Norwood, NJ), 15, 1 (1992), 37-72.}

This study examines the activity of storytelling at dinnertime in English-speaking, CaucasianAmerican families. The findings demonstrate that, through the process of story co-narration, family members draw upon and stimulate critical social, cognitive, and linguistic skills that underlie scientific and other scholarly discourse as they jointly construct, deconstruct, and reconstruct theories of everyday events. Each story is potentially a theory of a set of events in that it contains an explanation, which may then be overtly challenged and reworked by co-narrators. The data suggest that complex theory-building through storytelling is promoted by (and constitutive of) interlocutors' familiarity with one another and/or the narrative events. As such, long before children enter a classroom, everyday storytelling among familiars constitutes a commonplace medium for socialising perspectivetaking, critical thinking, and other intellectual skills that have been viewed as outcomes of formal schooling. 
92-472 Pearson, Bethyl A. and Lee, K. Samuel (Arizona State U.). Discourse structure of direction giving: effects of native/non-native speaker status and gender TESOL Quarterly (Washington, DC), 26, 1 (1992), 113-27.

This article tests the effects of native/non-native (NS/NNS) English-speaker status and gender on the structure of directions issued by native speakers of English. By extending the scope of earlier studies, this paper confirms that direction giving on the US university campus is highly conventionalised. At the same time, it argues that NS/NNS status and gender systematically influence a small range of linguistic choices in the exchange. Specifically, direction givers do make coordinated speech modifications, i.e., use certain features of 'foreigner register' to NNSs in this conversation type, but these adjustments do not match earlier findings. In addition, gender of both the direction giver and the direction seeker influence the structure and content of the discourse. The joint effects of these variables also play a relevant role. ESOL instruction can benefit, it is argued, by understanding the components of this discourse patterning.

\section{2-473 Rubin, Donald L. and Greene, Kathryn (U. of Georgia). Gender-typical} style in written language. Research in the Teaching of English (Urbana, III), 26, 1 (1992), 7-40.

Three lines of enquiry bear on the supposition that women's written language differs from men's in socially significant ways: (1) research on women's speech, (2) analyses of women's belles lettres, and (3) research on women's epistemology. This study was designed to test the supposition that male and female writing styles differ. College students' writing was subjected to a variety of lexical, syntactic, and text-level analyses for features that have been linked to writers' gender in previous research and theory. To determine whether gender differences were more pronounced in spontaneous expressive writing to an intimate audience, relative to revised instrumental writing to a distant audience, compositions representing these polar extremes were elicited. In addition to considering writers' bio- logical gender, the design of the study took into account measured gender role orientation as described in androgyny theory. Overall, the results of this study warrant the view that the writing of men and women is far more similar one to the other than different. Differences due to mode of discourse were more widespread than differences due to gender. Still, where male and female styles did diverge, they differed in predicted directions. For example, women used far more exclamation points than did men. In addition, women were more likely than men to acknowledge the legitimacy of opposing points of view. The findings of this study can inform discussions of instructional proposals regarding gender and writing.

\section{2-474 Sanders, Ted J. M. and others (Tilburg U., The Netherlands). Toward a} toxonomy of coherence relations. Discourse Processes (Norwood, NJ), 15, 1 (1992). $1-35$.

Understanding a discourse means constructing a coherent representation of that discourse. Inferring coherence relations, such as cause-consequence and claim-argument, is a necessary condition for a discourse representation to be coherent. Despite some descriptively fairly adequate proposals in the literature, there is still no theoretically satisfying account of the links that make a discourse coherent.

An adequate account of the relations establishing coherence has to be psychologically plausible, because coherence relations are ultimately cognitive relations. The authors propose a taxonomy that classifies coherence relations in terms of four cognitively salient primitives, such as the polarity of the relation and the pragmatic or semantic character of the link between the units.

A classification experiment using fragments of written discourse showed that the 12 classes of coherence relations distinguished in the taxonomy appear to be intuitively plausible and applicable. A second experiment investigating the use of connectives provided further evidence for the psychological salience of the taxonomic primitives and their relevance to the understanding of coherence relations. 
92-475 Schleppegrell, Mary J. Subordination and linguistic complexity. Discourse Processes (Newark, NJ), 15, 1 (1992), 117-131.

Subordination is frequently used as a measure of language complexity in educational and linguistic research. This article demonstrates, however, that in spoken discourse many clauses introduced by 'subordinating conjunctions' are not actually subordinate, whether subordination is considered a structural concept (the embedding of one clause within another) or an informational construct (main vs. subordinate ideas). This analysis suggests that the study of linguistic complexity must be informed by studies of spoken as well as written discourse and take account of the cultural and situational contexts of language use.

92-476 Smith, Janet S. (U. of California, Davis). Women in charge: politeness and directives in the speech of Japanese women. Language in Society (London), 21, 1 (1992), 59-82.

This article explores the linguistic practices of Japanese men and women giving directions to subordinates. Previous research on language and gender across a number of languages has equated the speech of women with powerlessness. The literature on Japanese women's speech would support this notion. It characterises Japanese female speech as soft, polite, indirect - in sum, as powerless. This presents problems for women who must command. 'The present study, an extension of the author's

previous work on Japanese female speech centred on women in more typically female roles, examines the directives of women in positions of authority in traditional and nontraditional domains and compares them with the directive forms chosen by men in similar positions. Explanations for the differences found are placed within the frameworks of a general theory of politeness and the culturally specific, gendered strategies for encoding politeness and authority in Japanese.

\section{2-477 Wood, Linda A. (U. of Guelph, Ontario, Canada) and Kroger, Rolf O. (U. of Toronto, Ontario, Canada). Politeness and forms of address. Journal of Language and Social Psychology (Clevedon. Avon), 10, 3 (1991), 145-68.}

This paper considers the politeness of forms of address in terms of Brown and Levinson's (1987) theory of politeness. A conceptual analysis of the elements of politeness is presented for six kinds of nominal address exchange and of the politeness expected for different categories of social relationship. These theoretical values are then compared to the politeness of address exchanges collected in a series of previous studies. The results of both the conceptual and empirical analyses are compatible with Brown and Levinson's assumption that nega- tive politeness outweighs positive politeness. However, it appears that the formula for deriving the weightiness of face-threatening acts and hence politeness requires revisions that would give greater weight to status than to solidarity and incorporate the interaction of status and solidarity. The authors discuss the plausibility of the claim that negative politeness is more polite than positive politeness and consider the implications of the results for understanding the expression of solidarity and the relationship of affect to politeness. 